\title{
On the chromatic number of the Erdős-Rényi orthogonal polarity graph
}

\author{
Xing Peng Michael Tait \\ Department of Mathematics \\ University of California at San Diego \\ La Jolla, California 92093-0112, U.S.A. \\ \{x2peng, mtait\}@ucsd.edu
}

\author{
Craig Timmons* \\ Department of Mathematics and Statistics \\ California State University Sacramento \\ Sacramento, CA 95819, U.S.A. \\ craig.timmons@csus.edu
}

Submitted: Dec 9, 2014; Accepted: Apr 24, 2015; Published: Apr 29, 2015

Mathematics Subject Classifications: 05C15, $05 \mathrm{C} 35$

\begin{abstract}
For a prime power $q$, let $E R_{q}$ denote the Erdős-Rényi orthogonal polarity graph. We prove that if $q$ is an even power of an odd prime, then $\chi\left(E R_{q}\right) \leqslant 2 \sqrt{q}+$ $O(\sqrt{q} / \log q)$. This upper bound is best possible up to a constant factor of at most 2. If $q$ is an odd power of an odd prime and satisfies some condition on irreducible polynomials, then we improve the best known upper bound for $\chi\left(E R_{q}\right)$ substantially. We also show that for sufficiently large $q$, every $E R_{q}$ contains a subgraph that is not 3 -chromatic and has at most 36 vertices.
\end{abstract}

Keywords: orthogonal polarity graphs; Turán number; forbidden subgraph; chromatic number

\section{Introduction}

Let $q$ be a prime power and let $V$ be a 3 -dimensional vector space over $\mathbb{F}_{q}$. Let $P G(2, q)$ be the projective geometry whose points are the 1-dimensional subspaces of $V$ and whose lines are the 2-dimensional subspaces of $V$. The Erdös-Rényi orthogonal polarity graph, denoted $E R_{q}$, is the graph whose vertices are the points of $P G(2, q)$, and distinct vertices $\left(x_{0}, x_{1}, x_{2}\right)$ and $\left(y_{0}, y_{1}, y_{2}\right)$ are adjacent if and only if $x_{0} y_{0}+x_{1} y_{1}+x_{2} y_{2}=0$. One obtains an isomorphic graph if the equation for adjacency is $x_{2} y_{0}+x_{0} y_{2}=x_{1} y_{1}$ (see [16]) and it is this definition of $E R_{q}$ that we will use. Throughout the paper, if $G$ is a graph and $S \subset V(G)$, we will use $G[S]$ to denote the subgraph induced by $S$.

These graphs were constructed independently by Brown [4], and Erdős, Rényi, and Sós [7] and have many applications to problems in extremal graph theory. The graph $E R_{q}$

\footnotetext{
*Research supported by CSU Sacramento Provost's Research Incentive Fund.
} 
has $q^{2}+q+1$ vertices, has $\frac{1}{2} q(q+1)^{2}$ edges, and has no 4-cycle as a subgraph. Füredi [9], [10] proved that for $q \geqslant 15$ a prime power, a graph with $q^{2}+q+1$ vertices and no 4-cycle has at most $\frac{1}{2} q(q+1)^{2}$ edges. The Erdős-Rényi graphs show that Füredi's upper bound is best possible. This is perhaps the most well-known application of $E R_{q}$ to extremal graph theory, but there are many others. The interested reader is referred to [14], [13], and [3] for applications of $E R_{q}$ to problems in hypergraph Turán theory, Ramsey theory, and structural graph theory.

Because of its important place in extremal graph theory, many researchers have studied the graph $E R_{q}$. Benny Sudakov posed the question of determining the independence number of $E R_{q}$ (see [17]), and it has since been investigated in several papers. Mubayi and Williford [16] proved that if $p$ is a prime, $n \geqslant 1$ is an integer, and $q=p^{n}$, then

$$
\alpha\left(E R_{q}\right) \geqslant \begin{cases}\frac{1}{2} q^{3 / 2}+\frac{1}{2} q+1 & \text { if } p \text { is odd and } n \text { is even } \\ \frac{120 q^{3 / 2}}{73 \sqrt{73}} & \text { if } p \text { is odd and } n \text { is odd } \\ \frac{q^{3 / 2}}{2 \sqrt{2}} & \text { if } p=2 \text { and } n \text { is odd } \\ q^{3 / 2}-q+q^{1 / 2} & \text { if } p=2 \text { and } n \text { is even. }\end{cases}
$$

An upper bound $\alpha\left(E R_{q}\right) \leqslant q^{3 / 2}+q^{1 / 2}+1$ can be obtained from Hoffman's bound. Therefore, the order of magnitude of $\alpha\left(E R_{q}\right)$ is $q^{3 / 2}$. Godsil and Newman refined the upper bound obtained from Hoffman's bound in [11]. Their result was then improved using the Lovász theta function in [6]. When $q$ is even, Hobart and Williford [12] used coherent configurations to provide upper bounds for the independence number of general orthogonal polarity graphs. When $q$ is an even square, the know upper bound and lower bound for $\alpha\left(E R_{q}\right)$ differ by at most 1 . In the case when $p$ is odd or when $p=2$ and $n$ is odd, it is still an open problem to determine an asymptotic formula for $\alpha\left(E R_{q}\right)$.

Since the independence number has been well-studied and its order of magnitude is known, it is natural to investigate the chromatic number of $E R_{q}$ which is closely related to $\alpha\left(E R_{q}\right)$. As $E R_{q}$ has $q^{2}+q+1$ vertices and $\alpha\left(E R_{q}\right)=\Theta\left(q^{3 / 2}\right)$, a lower bound for $\chi\left(E R_{q}\right)$ is $\frac{q^{2}+q+1}{\alpha\left(E R_{q}\right)} \geqslant q^{1 / 2}$. One may ask whether this lower bound actually gives the right order of magnitude of $\chi\left(E R_{q}\right)$. We confirm this for $q$ being an even power of an odd prime.

Theorem 1. If $q=p^{2 r}$ where $p$ is an odd prime and $r \geqslant 1$ is an integer, then

$$
\chi\left(E R_{q}\right) \leqslant 2 \sqrt{q}+O(\sqrt{q} / \log q) .
$$

This upper bound is within a factor of 2 of the lower bound $\chi\left(E R_{q}\right) \geqslant \frac{q^{2}+q+1}{\alpha\left(E R_{q}\right)} \geqslant$ $q^{1 / 2}$. Any improvement in the coefficient of $q^{1 / 2}$ would give an improvement to the best known lower bound on the independence number of $E R_{q}$ from [16]. The lower order term $O\left(q^{1 / 2} / \log q\right)$ is obtained using probabilistic methods [2] and while the implied constant is absolute, we have not made an effort to compute it. By using Brooks' Theorem instead 
of the result of Alon et. al. [2], we obtain the upper bound $\chi\left(E R_{q}\right) \leqslant 4 q^{1 / 2}+1$ for all $q=p^{2 r}$ where $r \geqslant 1$ is an integer and $p$ is an odd prime.

When $q$ is not an even power, we first prove the following general theorem.

Theorem 2. Let $q$ be an odd power of an odd prime and let $r \geqslant 1$ be an integer. If there is a $\mu \in \mathbb{F}_{q}$ such that $x^{2 r+1}-\mu$ is irreducible in $\mathbb{F}_{q}[x]$, then

$$
\chi\left(E R_{q^{2 r+1}}\right) \leqslant \frac{2 r+5}{3} q^{\frac{4 r}{3}+1}+(2 r+1) q^{r+1}+1 .
$$

Given an odd integer $2 r+1 \geqslant 3$, there are infinitely many primes $p$ for which there is a $\mu \in \mathbb{F}_{q}$ such that $x^{2 r+1}-\mu \in \mathbb{F}_{q}[x]$ is irreducible (see Section 5 for more details), where $q$ is an arbitrary odd power of $p$. Our method can also be used to prove that if $q$ is a power of any odd prime, then

$$
\chi\left(E R_{q^{3}}\right) \leqslant 6 q^{2}+1 .
$$

Here we do not need the existence of an irreducible polynomial $x^{3}-\mu \in \mathbb{F}_{q}[x]$.

For $q$ is an odd power of an odd prime, we have the following corollary.

Corollary 3. Let $q=p^{s}$ for an odd prime $p$ and an odd integer $s \geqslant 3$. If $t>1$ is the smallest divisor of $s$ such that $x^{t}-\mu$ is irreducible in $\mathbb{F}_{p^{s / t}}[x]$ for some $\mu \in \mathbb{F}_{p^{s / t}}$, then

$$
\chi\left(E R_{q}\right) \leqslant \frac{t+4}{3} p^{s(2 t+1) / 3 t}+t p^{(t+1) / 2}+1 .
$$

We encountered difficulties in extending this upper bound to the general case. In particular, when $p$ is a prime, we have not been able to improve the upper bound $\chi\left(E R_{p}\right)=$ $O(p / \log p)$ which is obtained by applying the main result of [2].

Conjecture 4. Let $p$ be an odd prime. For any integer $r \geqslant 0$,

$$
\chi\left(E R_{p^{2 r+1}}\right)=O\left(p^{r+1 / 2}\right) .
$$

Instead of working with $E R_{q}$, we work with a related graph that is a bit more suitable for our computations.

Definition 5. Let $q$ be a power of an odd prime and $A=\left\{\left(a, a^{2}\right): a \in \mathbb{F}_{q}\right\}$. Let $G_{q}$ be the graph with vertex set $\mathbb{F}_{q} \times \mathbb{F}_{q}$, and distinct vertices $\left(x_{1}, x_{2}\right)$ and $\left(y_{1}, y_{2}\right)$ are adjacent if and only if

$$
\left(x_{1}, x_{2}\right)+\left(y_{1}, y_{2}\right) \in A .
$$

Let $G_{q}^{\circ}$ be the graph obtained from $G_{q}$ by adding loops to all vertices $\left(x_{1}, x_{2}\right)$ for which $\left(x_{1}, x_{2}\right)+\left(x_{1}, x_{2}\right) \in A$. Vinh [18] proved that the graph $G_{q}^{\circ}$ is a $\left(q^{2}, q, \sqrt{2 q}\right)$-graph. Recall an $(n, d, \lambda)$ graph is an $n$-vertex $d$-regular graph whose second eigenvalue $\max \left\{\left|\lambda_{2}\right|,\left|\lambda_{n}\right|\right\}$ is at most $\lambda$. Vinh used the fact that $G_{q}^{\circ}$ is a $\left(q^{2}, q, \sqrt{2 q}\right)$-graph to count solutions to $x_{1}+x_{2}=\left(x_{3}+x_{4}\right)^{2}$ where $\left(x_{1}, x_{3}\right) \subset B,\left(x_{2}, x_{4}\right) \subset C$, and $B, C \subset \mathbb{F}_{q}^{2}$. For similar results that are obtained using techniques from combinatorial number theory, see [5]. We prove that $G_{q}$ is isomorphic to an induced subgraph of the Erdös-Rényi orthogonal polarity graph. 
Theorem 6. If $q$ is a power of an odd prime, then the graph $G_{q}$ is isomorphic to an induced subgraph of $E R_{q}$.

In the course of proving Theorem 6 we will show how to obtain $E R_{q}$ from $G_{q}$ by adding vertices and edges to $G_{q}$. This will allow us to translate upper bounds on $\chi\left(G_{q}\right)$ to upper bounds on $\chi\left(E R_{q}\right)$.

In addition to finding a proper coloring of $E R_{q}$, we also investigate proper colorings of small subgraphs of $E R_{q}$. In particular, we obtain the following result concerning small subgraphs of $E R_{q}$ that are not 3-colorable.

Theorem 7. If $q$ is a sufficiently large odd prime power, then $E R_{q}$ contains a subgraph $H$ with at most 36 vertices and $\chi(H) \geqslant 4$.

If $\mathcal{F}$ is a family of graphs, we say that a graph $G$ is $\mathcal{F}$-free if $G$ does not contain a subgraph isomorphic to a graph in $\mathcal{F}$. One of the most well-studied problems in extremal graph theory is to determine the maximum number of edges in an $\mathcal{F}$-free graph with $n$ vertices, and then describe the extremal $\mathcal{F}$-free graphs. When $\mathcal{F}$ contains bipartite graphs, the structure of extremal $\mathcal{F}$-free graphs is not very well understood and there are few general results. A notable exception is when $\mathcal{F}=\left\{C_{4}\right\}$. A result of Füredi [10] states that when $q \geqslant 15$ is a prime power, an extremal $C_{4}$-free graph with $q^{2}+q+1$ vertices is an orthogonal polarity graph of a projective plane (see Section 5 for the definition of an orthogonal polarity graph).

Let $\mathcal{C}^{r}$ be the family of graphs with chromatic number $r$, and $\mathcal{C}_{k}^{r}$ be the family of graphs with at most $k$ vertices and chromatic number $r$. Theorem 7 is motivated by the following problem of Allen, Keevash, Sudakov, and Verstraëte [1].

Problem 8 (Allen, et al. [1]). Let $\mathcal{F}$ be a family of bipartite graphs. Determine if there is an integer $k$ such that

$$
\operatorname{ex}\left(n, \mathcal{F} \cup \mathcal{C}_{k}^{r}\right) \sim \operatorname{ex}\left(n, \mathcal{F} \cup \mathcal{C}^{r}\right) .
$$

When considering Problem 8 , a question that arises is if every extremal $\mathcal{F}$-free $n$-vertex graph (here $n$ is tending to infinity) must contain some member of $\mathcal{C}_{k}^{r}$ ? In other words, does forbidding $\mathcal{C}_{k}^{r}$ actually have an effect on extremal $\mathcal{F}$-free graphs. By Theorem 7 , one cannot take $E R_{q}$ to obtain a lower bound on the Turán number $\operatorname{ex}\left(n,\left\{C_{4}\right\} \cup \mathcal{C}_{k}^{3}\right)$ for $k \geqslant 36$ without modifying $E R_{q}$ in some way. It seems likely that for any integer $r \geqslant 5$, there exists integers $q_{r}$ and $f(r)$ such that for any $q \geqslant q_{r}$, the graph $E R_{q}$ contains a subgraph with at most $f(r)$ vertices and chromatic number at least $r$.

In Section 2 we prove Theorem 6. In Section 3 we prove Theorems 1 and 2. In Section 4 we prove Theorem 7 . Section 5 contains some concluding remarks.

\section{Proof of Theorem 6}

Let $q$ be a power of an odd prime power and $A=\left\{\left(a, a^{2}\right): a \in \mathbb{F}_{q}\right\}$. Let $\mathbb{F}_{q}=\left\{b_{1}, \ldots, b_{q}\right\}$ and assume that $b_{q}=0$. Let $F=\left\{b_{q}\right\} \times \mathbb{F}_{q}$. Then $F$ is a subgroup of $\mathbb{F}_{q}^{2}$ and we let

$$
F_{i}=F+\left(b_{i}, 0\right)
$$


be the cosets of $F$ where $F_{q}=F$. Add new vertices $z_{1}, \ldots, z_{q}, y$ to $G_{q}$. Make $z_{i}$ adjacent to all vertices in $F_{i}$, and make $y$ adjacent to each $z_{i}$. Call this graph $H_{q}$. Observe that $G_{q}$ is an induced subgraph of $H_{q}$. We define an isomorphism $\phi$ from $H_{q}$ to $E R_{q}$ as follows.

1. For any $b_{j} \in \mathbb{F}_{q}$, let $\phi\left(\left(0, b_{j}\right)\right)=\left(1,0,2^{-1} b_{j}\right)$.

2. For any $b_{i}, b_{j} \in \mathbb{F}_{q}$ with $b_{i} \neq 0$, let $\phi\left(\left(b_{i}, b_{j}\right)\right)=\left(1, b_{i}, 2^{-1}\left(b_{j}-b_{i}^{2}\right)\right)$.

3. Let $\phi(y)=(0,0,1)$ and $\phi\left(z_{i}\right)=\left(0,1, b_{i}\right)$ for $1 \leqslant i \leqslant q$.

We will show that $\phi$ is an isomorphism by considering the different types of vertices in $H_{q}$. Recall that the rule for adjacency in $E R_{q}$ is that $\left(x_{0}, x_{1}, x_{2}\right)$ is adjacent to $\left(y_{0}, y_{1}, y_{2}\right)$ if and only if $x_{0} y_{2}+x_{2} y_{0}=x_{1} y_{1}$.

Case 1: Vertices of type $\left(0, b_{j}\right)$.

Let $b_{j} \in \mathbb{F}_{q}$. In $H_{q}$, the neighborhood of $\left(0, b_{j}\right)$ is $\left\{z_{q}\right\} \cup\left\{\left(x, x^{2}-b_{j}\right): x \in \mathbb{F}_{q}\right\}$. In $E R_{q}$, the neighborhood of $\left(1,0,2^{-1} b_{j}\right)$ is

$$
\{(0,1,0)\} \cup\left\{\left(1, x,-2^{-1} b_{j}\right): x \in \mathbb{F}_{q}\right\} .
$$

By definition, $\phi\left(\left(0,-b_{j}\right)\right)=\left(1,0,-2^{-1} b_{j}\right)$ and for $x \neq 0$,

$$
\phi\left(\left(x, x^{2}-b_{j}\right)\right)=\left(1, x, 2^{-1}\left(x^{2}-b_{j}-x^{2}\right)\right)=\left(1, x,-2^{-1} b_{j}\right) .
$$

This shows that (1) coincides with the set

$$
\left\{\phi\left(z_{q}\right)\right\} \cup\left\{\phi\left(\left(x, x^{2}-b_{j}\right)\right): x \in \mathbb{F}_{q}\right\} .
$$

We conclude that for any $b_{j} \in \mathbb{F}_{q},\left(0, b_{j}\right)$ is adjacent to $u$ in $H_{q}$ if and only if $\phi\left(\left(0, b_{j}\right)\right)$ is adjacent to $\phi(u)$ in $E R_{q}$.

Case 2: Vertices of type $\left(b_{i}, b_{j}\right)$ with $b_{i} \neq 0$.

Let $b_{i}, b_{j} \in \mathbb{F}_{q}$ with $b_{i} \neq 0$. In $H_{q}$, the neighborhood of $\left(b_{i}, b_{j}\right)$ is

$$
\left\{z_{i}\right\} \cup\left\{\left(x-b_{i}, x^{2}-b_{j}\right): x \in \mathbb{F}_{q}\right\} .
$$

In $E R_{q}$, the neighborhood of $\left(1, b_{i}, 2^{-1}\left(b_{j}-b_{i}^{2}\right)\right)$ is

$$
\left\{\left(0,1, b_{i}\right)\right\} \cup\left\{\left(1, x, x b_{i}-2^{-1}\left(b_{j}-b_{i}^{2}\right)\right): x \in \mathbb{F}_{q}\right\} .
$$

We have $\phi\left(z_{i}\right)=\left(0,1, b_{i}\right)$ and

$$
\phi\left(\left(b_{i}-b_{i}, b_{i}^{2}-b_{j}\right)\right)=\left(1,0,-2^{-1}\left(b_{j}-b_{i}^{2}\right)\right) .
$$

For $y \neq b_{i}$,

$$
\begin{aligned}
\phi\left(\left(y-b_{i}, y^{2}-b_{j}\right)\right) & =\left(1, y-b_{i}, 2^{-1}\left(y^{2}-b_{j}-\left(y-b_{i}\right)^{2}\right)\right) \\
& =\left(1, y-b_{i}, y b_{i}-2^{-1}\left(b_{j}+b_{i}^{2}\right)\right) .
\end{aligned}
$$


If we take $x=y-b_{i}$ in (2), we obtain

$$
\left(1, y-b_{i}, y b_{i}-2^{-1}\left(b_{j}+b_{i}^{2}\right)\right)
$$

using the fact that $2^{-1}-1=-2^{-1}$. We conclude that for any $b_{i}, b_{j} \in \mathbb{F}_{q}$ with $b_{i} \neq 0$, $\left(b_{i}, b_{j}\right)$ is adjacent to $u$ in $H_{q}$ if and only if $\phi\left(\left(b_{i}, b_{j}\right)\right)$ is adjacent to $\phi(u)$ in $E R_{q}$.

Case 3: Vertices of type $z_{i}$.

Let $1 \leqslant i \leqslant q$ and consider $z_{i}$. The neighborhood of $z_{i}$ is $\{y\} \cup\left\{\left(b_{i}, x\right): x \in \mathbb{F}_{q}\right\}$. In $E R_{q}$, the neighborhood of $\left(0,1, b_{i}\right)$ is

$$
\{(0,0,1)\} \cup\left\{\left(1, b_{i}, x\right): x \in \mathbb{F}_{q}\right\}=\{\phi(y)\} \cup\left\{\left(1, b_{i}, x\right): x \in \mathbb{F}_{q}\right\} .
$$

If $i=q$, then $\phi((0, y))=\left(1,0,2^{-1} y\right)$. If $i \neq q$, then $\phi\left(\left(b_{i}, y\right)\right)=\left(1, b_{i}, 2^{-1}\left(y-b_{i}^{2}\right)\right)$. As $y$ ranges over $\mathbb{F}_{q}$, we obtain $\left(1, b_{i}, x\right)$ for all $x \in \mathbb{F}_{q}$.

We have not checked the neighborhood condition for $y \in V\left(H_{q}\right)$ but since we have considered all other vertices, this is not necessary.

\section{Proof of Theorems 1 and 2}

Throughout this section $p$ is an odd prime and $q$ is a power of $p$. The set $\mathbb{F}_{q}^{*}$ consisting of the nonzero elements of $\mathbb{F}_{q}$ can be partitioned into two sets $\mathbb{F}_{q}^{+}$and $\mathbb{F}_{q}^{-}$where

$$
a \in \mathbb{F}_{q}^{+} \text {if and only if }-a \in \mathbb{F}_{q}^{-} .
$$

Observe that the vertices $\left(x_{1}, x_{2}\right)$ and $\left(y_{1}, y_{2}\right)$ are adjacent in $G_{q}$ if and only if $x_{1}+y_{1}=a$ and $x_{2}+y_{2}=a^{2}$ for some $a \in \mathbb{F}_{q}$. This is equivalent to $\left(x_{1}+y_{1}\right)^{2}=x_{2}+y_{2}$. It is often this relation that we will use in our calculations.

Lemma 9. (i) If $\mathbb{F}_{q^{2}}=\left\{a \theta+b: a, b \in \mathbb{F}_{q}\right\}$ for some $\theta \in \mathbb{F}_{q^{2}} \backslash \mathbb{F}_{q}$, then both

$$
\left\{(x, y \theta+z): x, z \in \mathbb{F}_{q}, y \in \mathbb{F}_{q}^{+}\right\} \text {and }\left\{(x, y \theta+z): x, z \in \mathbb{F}_{q}, y \in \mathbb{F}_{q}^{-}\right\}
$$

are independent sets in $G_{q}$.

(ii) If $t \geqslant 3$ is odd and $\mathbb{F}_{q^{t}}=\left\{a_{0}+\cdots+a_{t-1} \theta^{t-1}: a_{i} \in \mathbb{F}_{q}\right\}$ for some $\theta \in \mathbb{F}_{q^{t}}$, then both

$$
\left\{\left(x_{0}+\cdots+x_{(t-3) / 2} \theta^{\frac{t-3}{2}}, y_{0}+\cdots+y_{t-1} \theta^{t-1}\right): x_{i}, y_{j} \in \mathbb{F}_{q}, y_{t-1} \in \mathbb{F}_{q}^{+}\right\}
$$

and

$$
\left\{\left(x_{0}+\cdots+x_{(t-3) / 2} \theta^{\frac{t-3}{2}}, y_{0}+\cdots+y_{t-1} \theta^{t-1}\right): x_{i}, y_{j} \in \mathbb{F}_{q}, y_{t-1} \in \mathbb{F}_{q}^{-}\right\}
$$

are independent sets in $G_{q}$.

Proof. We prove the first case of (i) as the proofs of the remaining statements are very similar. Suppose $\left(x_{1}, y_{1} \theta+z_{1}\right)$ and $\left(x_{2}, y_{2} \theta+z_{2}\right)$ are vertices in $G_{q}$ with $x_{1}, x_{2}, z_{1}, z_{2} \in \mathbb{F}_{q}$ and $y_{1}, y_{2} \in \mathbb{F}_{q}^{+}$. Then $\left(x_{1}+x_{2}\right)^{2} \in \mathbb{F}_{q}$ but $\left(y_{1}+y_{2}\right) \theta+\left(z_{1}+z_{2}\right) \notin \mathbb{F}_{q}$ since $y_{1}+y_{2} \neq 0$. Therefore, the vertices $\left(x_{1}, y_{1} \theta+z_{1}\right)$ and $\left(x_{2}, y_{2} \theta+z_{2}\right)$ are not adjacent. 
Lemma 10. For any $k \in \mathbb{F}_{q}^{*}$, the maps $\psi_{k}, \phi_{k}: V\left(G_{q}\right) \rightarrow V\left(G_{q}\right)$ given by

$$
\psi_{k}((x, y))=\left(x+k, y+4 k x+2 k^{2}\right) \text { and } \phi_{k}((x, y))=\left(k x, k^{2} y\right)
$$

are automorphisms of $G_{q}$.

Proof. Let $k \in \mathbb{F}_{q}^{*}$. Suppose $\left(x_{1}, x_{2}\right)$ is adjacent to $\left(y_{1}, y_{2}\right)$ so that $\left(x_{1}+y_{1}\right)^{2}=x_{2}+y_{2}$. In this case,

$$
\begin{aligned}
\left(x_{1}+k+y_{1}+k\right)^{2} & =\left(x_{1}+y_{1}\right)^{2}+4 k x_{1}+4 k y_{1}+4 k^{2} \\
& =\left(x_{2}+4 k x_{1}+2 k^{2}\right)+\left(y_{2}+4 k y_{1}+2 k^{2}\right) .
\end{aligned}
$$

This shows that $\left(x_{1}+k, x_{2}+4 k x_{1}+2 k^{2}\right)$ is adjacent to $\left(y_{1}+k, y_{2}+4 k y_{1}+2 k^{2}\right)$. Conversely, if $\left(x_{1}+k, x_{2}+4 k x_{1}+2 k^{2}\right)$ is adjacent to $\left(y_{1}+k, y_{2}+4 k y_{1}+2 k^{2}\right)$, then it must be the case that $\left(x_{1}+y_{1}\right)^{2}=x_{2}+y_{2}$ and so $\left(x_{1}, y_{1}\right)$ is adjacent to $\left(x_{2}, y_{2}\right)$.

To show that $\phi_{k}$ is an isomorphism it is enough to observe that $\left(x_{1}+y_{1}\right)^{2}=x_{2}+y_{2}$ is equivalent to $\left(k x_{1}+k y_{1}\right)^{2}=k^{2} x_{2}+k^{2} y_{2}$.

\section{$3.1 \quad q$ a square}

In this section we prove the following.

Theorem 11. Let $q$ be a power of an odd prime. The chromatic number of $G_{q^{2}}$ satisfies

$$
\chi\left(G_{q^{2}}\right) \leqslant 2 q+O(q / \log q) .
$$

Proof. Let $\theta$ be a root of an irreducible quadratic polynomial in $\mathbb{F}_{q}[x]$ so that $\mathbb{F}_{q^{2}}=$ $\left\{a \theta+b: a, b, \in \mathbb{F}_{q}\right\}$. Assume that $\theta^{2}=\mu_{1} \theta+\mu_{0}$ where $\mu_{0}, \mu_{1} \in \mathbb{F}_{q}$. Let $I^{+}=\{(x, y \theta+z)$ : $\left.x, z \in \mathbb{F}_{q}, y \in \mathbb{F}_{q}^{+}\right\}, I^{-}=\left\{(x, y \theta+z): x, z \in \mathbb{F}_{q}, y \in \mathbb{F}_{q}^{-}\right\}$, and $J=I^{+} \cup I^{-}$. By Lemma $9, J$ is the union of two independent sets and so $\chi\left(G_{q^{2}}[J]\right) \leqslant 2$. Let

$$
S=\bigcup_{k \in \mathbb{F}_{q}} \psi_{k \theta}(J)
$$

By Lemma 10, each $\psi_{k \theta}$ is an isomorphism and so $\chi\left(G_{q^{2}}[S]\right) \leqslant 2 q$. Let $X=V\left(G_{q^{2}}\right) \backslash S$. Since $\mathbb{F}_{q}^{+} \cup \mathbb{F}_{q}^{-}=\mathbb{F}_{q}^{*}$, we can write

$$
S=\left\{\left(x+k \theta, y \theta+z+4 k \theta x+2 k^{2} \theta^{2}\right): x, k, y, z \in \mathbb{F}_{q}, y \neq 0\right\} .
$$

Given a vertex $(s, t) \in V\left(G_{q^{2}}\right)$, say with $s=s_{0}+s_{1} \theta$ and $t=t_{0}+t_{1} \theta$, we can take $x=s_{0}$ and $k=s_{1}$ to obtain

$$
\left\{\left(s_{0}+s_{1} \theta, y \theta+z+4 s_{1} s_{0} \theta+2 s_{1}^{2}\left(\mu_{1} \theta+\mu_{0}\right)\right): y, z \in \mathbb{F}_{q}, y \neq 0\right\} \subset S .
$$

The second coordinate in the above subset of $S$ simplifies to

$$
\left(z+2 s_{1}^{2} \mu_{0}\right)+\left(y+4 s_{1} s_{0}+2 s_{1}^{2} \mu_{1}\right) \theta .
$$


We can choose $z=t_{0}-2 s_{1}^{2} \mu_{0}$ and as long as $t_{1} \neq 4 s_{1} s_{0}+2 s_{1}^{2} \mu_{1}$, we can take $y=$ $t_{1}-4 s_{1} s_{0}-2 s_{1}^{2} \mu_{1}$. Otherwise, $t_{1}=4 s_{1} s_{0}+2 s_{1}^{2} \mu_{1}$ and so

$$
X=\left\{\left(s_{0}+s_{1} \theta, t_{0}+\left(4 s_{1} s_{0}+2 s_{1}^{2} \mu_{1}\right) \theta\right): s_{0}, s_{1}, t_{0} \in \mathbb{F}_{q}\right\} .
$$

Partition $X$ into $q$ sets $X_{s}$ where $s \in \mathbb{F}_{q}$ and

$$
X_{s}=\left\{\left(s \theta+s_{2},\left(2 s^{2} \mu_{1}+4 s s_{2}\right) \theta+t_{2}: s_{2}, t_{2} \in \mathbb{F}_{q}\right\} .\right.
$$

Claim 1: For any $s \in \mathbb{F}_{q}, \Delta\left(G_{q^{2}}\left[X_{s}\right]\right) \leqslant q$.

Let $s \in \mathbb{F}_{q}$. A pair of vertices

$$
\left(s \theta+s_{2},\left(2 s^{2} \mu_{1}+4 s s_{2}\right) \theta+t_{2}\right) \text { and }\left(s \theta+u_{2},\left(2 s^{2} \mu_{1}+4 s u_{2}\right) \theta+v_{2}\right),
$$

both in $X_{s}$, are adjacent if and only if $4 s^{2} \mu_{0}+\left(s_{2}+u_{2}\right)^{2}=t_{2}+v_{2}$. If $s_{2}$ and $t_{2}$ are fixed, then there are $q$ choices for $u_{2}$ and once $u_{2}$ is fixed, $v_{2}$ is determined. Therefore, the maximum degree of $G_{q^{2}}\left[X_{s}\right]$ is $q$.

Claim 2: $\Delta\left(G_{q^{2}}[X]\right) \leqslant 2 q-1$.

By Claim 1, a vertex in $X_{s}$ has at most $q$ other neighbors in $X_{s}$. Let $s, t \in \mathbb{F}_{q}$ where $s \neq t$. The vertex $\left(s \theta+s_{2},\left(2 s^{2} \mu_{1}+4 s s_{2}\right) \theta+t_{2}\right) \in X_{s}$ is adjacent to the vertex $\left(t \theta+u_{2},\left(2 t^{2} \mu_{1}+4 t u_{2}\right) \theta+v_{2}\right) \in X_{t}$ if and only if

$$
\mu_{1}\left(s^{2}+2 s t+t^{2}\right)+2(s+t) s_{2}+2(s+t) u_{2}=\mu_{1}\left(2 s^{2}+2 t^{2}\right)+4 s s_{2}+4 t u_{2}
$$

and

$$
(s+t)^{2} \mu_{0}+\left(s_{2}+u_{2}\right)^{2}=t_{2}+v_{2} .
$$

Equation (3) can be rewritten as

$$
\mu_{1}(s-t)^{2}=2(t-s) s_{2}+2(s-t) u_{2} .
$$

Thus if $s_{2}$ and $t_{2}$ are fixed, then (5) and (4) determine $u_{2}$ and $v_{2}$ since $2(s-t) \neq 0$. This shows that a vertex in $X_{s}$ has exactly one neighbor in $X_{t}$ whenever $s \neq t$. Namely, given the vertex $\left(s \theta+s_{2},\left(2 s^{2} \mu_{1}+4 s s_{2}\right) \theta+t_{2}\right) \in X_{s}$, its unique neighbor in $X_{t}$ where $t \neq s$ is $\left(t \theta+u_{2},\left(2 t^{2} \mu_{1}+4 t u_{2}\right) \theta+v_{2}\right)$ where

$$
u_{2}=2^{-1} \mu_{1}(s-t)+s_{2} \text { and } v_{2}=(s+t)^{2} \mu_{0}+\left(2 s_{2}+2^{-1} \mu_{1}(s-t)\right)^{2}-t_{2} .
$$

We conclude that a vertex $x \in X$ has at most $q$ neighbors in $X_{s}$ when $x \in X_{s}$, and one neighbor in each $X_{t}$ for $t \neq s$. Since $X=\cup_{s \in \mathbb{F}_{q}} X_{s}$, we have proved Claim 2 .

Alon, Krivelevich, and Sudakov [2] proved that any graph with maximum degree $d$ with the property that the neighborhood of every vertex contains at most $d^{2} / f$ edges has chromatic number at most $c(d / \log f)$ where $c$ is an absolute constant. A $C_{4}$-free graph with maximum degree $d$ has the property that the neighborhood of every vertex contains at most $d / 2$ edges. Applying the result of $[2]$ to $G_{q^{2}}[X]$, we obtain $\chi\left(G_{q^{2}}[X]\right)=O(2 q / \log q)$. Combining this coloring with our coloring of $S$, we obtain a proper coloring of $G_{q^{2}}$ with $2 q+O(q / \log q)$ colors. 
To obtain a coloring of $E R_{q^{2}} \cong H_{q^{2}}$, we only need one additional color for the vertices $z_{1}, \ldots, z_{q^{2}}, y$. The vertices $z_{1}, \ldots, z_{q^{2}}$ form an independent set in $H_{q^{2}}$ and so we use one new color on these vertices. The vertex $y$ has no neighbors in $G_{q^{2}}$ and so we may use any one of the $2 q+O(q / \log q)$ colors used to $G_{q^{2}}$ to color $y$. This proves Theorem 1 .

\section{$3.2 q$ not a square}

In this subsection we prove the following result.

Theorem 12. Let $q$ be a power of an odd prime. If $r \geqslant 1$ and for some $\mu \in \mathbb{F}_{q}$, the polynomial $x^{2 r+1}-\mu \in \mathbb{F}_{q}[x]$ is irreducible, then

$$
\chi\left(G_{q^{2 r+1}}\right) \leqslant \frac{2 r+5}{3} q^{\frac{4 r}{3}+1}+(2 r+1) q^{r+1} .
$$

Proof. Let $t=2 r+1 \geqslant 3$ be an odd integer. Suppose there is a $\mu \in \mathbb{F}_{q}^{*}$ such that the polynomial $x^{t}-\mu \in \mathbb{F}_{q}[x]$ is irreducible. Let $\theta$ be a root of $x^{t}-\mu$ in an extension field of $\mathbb{F}_{q}$. We may view $\theta$ as an element of $\mathbb{F}_{q^{t}}$ and $\left\{1, \theta, \ldots, \theta^{2 r}\right\}$ is a basis for $\mathbb{F}_{q^{t}}$ over $\mathbb{F}_{q}$. For $2 r+1 \leqslant l \leqslant 6 r+3$

$$
\theta^{l}= \begin{cases}\mu \theta^{l-2 r-1} & \text { if } 2 r+1 \leqslant l<4 r+2 \\ \mu^{2} \theta^{l-4 r-2} & \text { if } 4 r+2 \leqslant l<6 r+3\end{cases}
$$

This identity will be used frequently throughout this subsection. Define

$$
I^{+}=\left\{\left(x_{0}+x_{1} \theta+\cdots+x_{r-1} \theta^{r-1}, y_{0}+\cdots+y_{2 r} \theta^{2 r}\right): x_{i}, y_{j} \in \mathbb{F}_{q}, y_{2 r} \in \mathbb{F}_{q}^{+}\right\}
$$

and

$$
I^{-}=\left\{\left(x_{0}+x_{1} \theta+\cdots+x_{r-1} \theta^{r-1}, y_{0}+\cdots+y_{2 r} \theta^{2 r}\right): x_{i}, y_{j} \in \mathbb{F}_{q}, y_{2 r} \in \mathbb{F}_{q}^{-}\right\} .
$$

By Lemma 9, both $I^{+}$and $I^{-}$are independent sets. Let $J=I^{+} \cup I^{-}$. Since $J$ is the union of two independent sets, $\chi\left(G_{q^{t}}[J]\right) \leqslant 2$. For $k \in \mathbb{F}_{q^{t}}$, the map $\psi_{k}((x, y))=$ $\left(x+k, y+4 k x+2 k^{2}\right)$ is an isomorphism of $G_{q^{t}}$ by Lemma 10. Let

$$
S=\bigcup_{\left(x_{r}, \ldots, x_{2 r}\right) \in \mathbb{F}_{q}^{r+1}} \psi_{x_{r} \theta^{r}+\cdots+x_{2 r} \theta^{2 r}}(J) .
$$

We properly color the vertices of $S$ with at most $2 q^{r+1}$ colors. Let $X=\mathbb{F}_{q^{t}}^{2} \backslash S$. It remains to color the vertices in $X$. To do this, we will proceed as follows. By Lemma 10, for any $k \in \mathbb{F}_{q^{t}}^{*}$, the map $\phi_{k}((x, y))=\left(k x, k^{2} y\right)$ is an isomorphism of $G_{q^{t}}$. Let $1 \leqslant l \leqslant 2 r$ and consider $\phi_{\theta^{l}}(X)$. Let $Y_{l}=S \cap \phi_{\theta^{l}}(X)$. The graph $G_{q^{t}}\left[Y_{l}\right]$ is isomorphic to a subgraph of $G_{q^{t}}[S]$. We have shown that $\chi\left(G_{q^{t}}[S]\right) \leqslant 2 q^{r+1}$ and so $\chi\left(G_{q^{t}}\left[Y_{l}\right]\right) \leqslant 2 q^{r+1}$ for any $1 \leqslant l \leqslant 2 r$. Therefore, we can properly color the vertices in

$$
\phi_{\theta^{l}}^{-1}\left(Y_{l}\right)=\phi_{\theta^{l}}^{-1}(S) \cap X
$$


with at most $2 q^{r+1}$ colors. This gives a proper coloring that uses at most $(2 r+1) 2 q^{r+1}$ colors. The only vertices that have not been colored are those that are in the set

$$
Z:=X \cap \phi_{\theta}(X) \cap \phi_{\theta^{2}}(X) \cap \cdots \cap \phi_{\theta^{2 r}}(X) .
$$

We are now going to show that if $\left(s_{0}+\cdots+s_{2 r} \theta^{2 r}, t_{0}+\cdots+t_{2 r} \theta^{2 r}\right) \in Z$, then each $t_{i}$ is determined by $s_{0}, \ldots, s_{2 r}$. This will allow us to prove an upper bound on the maximum degree of $G_{q^{t}}[Z]$ and we can then color $G_{q^{t}}[Z]$ by applying Brooks' Theorem.

We will use the following notation for the rest of this section. If $s \in \mathbb{F}_{q^{t}}$, then $s_{0}, \ldots, s_{2 r}$ will be the coefficients of $s$ in the unique representation $s=s_{0}+s_{1} \theta+\cdots+s_{2 r} \theta^{2 r}$ where $s_{i} \in \mathbb{F}_{q}$. Given a $2 r+1$-tuple $\left(z_{0}, z_{1}, \ldots, z_{2 r}\right) \in \mathbb{F}_{q}^{2 r+1}$, define

$$
\alpha\left(z_{0}, z_{1}, \ldots, z_{2 r}\right)=2 z_{r}^{2}+4 \sum_{j=0}^{r-1} z_{j} z_{2 r-j} .
$$

Claim 1: If $\left(s_{0}+\cdots+s_{2 r} \theta^{2 r}, t_{0}+\cdots+t_{2 r} \theta^{2 r}\right) \in X$, then

$$
t_{2 r}=\alpha\left(s_{0}, s_{1}, \ldots, s_{2 r}\right)
$$

Proof of Claim 1. A vertex in $S$ is of the form

$$
\begin{aligned}
& \left(x_{0}+\cdots+x_{r-1} \theta^{r-1}+x_{r} \theta^{r}+\cdots+x_{2 r} \theta^{2 r}, y_{0}+\cdots+y_{2 r} \theta^{2 r}\right. \\
& \left.\quad+4\left(x_{r} \theta^{r}+\cdots+x_{2 r} \theta^{2 r}\right)\left(x_{0}+\cdots+x_{r-1} \theta^{r-1}\right)+2\left(x_{r} \theta^{r}+\cdots+x_{2 r} \theta^{2 r}\right)^{2}\right)
\end{aligned}
$$

for some $x_{i}, y_{j} \in \mathbb{F}_{q}$, and $y_{2 r} \in \mathbb{F}_{q}^{+} \cup \mathbb{F}_{q}^{-}=\mathbb{F}_{q}^{*}$. The coefficient of $\theta^{2 r}$ in the second coordinate is

$$
y_{2 r}+2 x_{r}^{2}+4 \sum_{j=0}^{r-1} x_{j} x_{2 r-j} .
$$

Thus, given any vertex $(s, t) \in \mathbb{F}_{q^{t}}^{2}$, we have that $(s, t) \in S$ unless

$$
t_{2 r}=2 s_{r}^{2}+\sum_{j=0}^{r-1} s_{j} s_{2 r-j} .
$$

Claim 2: If $1 \leqslant l \leqslant 2 r$ and $(s, t) \in X \cap \phi_{\theta^{l}}(X)$, then

$$
t_{2 l-1}=\mu \alpha\left(s_{l}, s_{l+1}, \ldots, s_{2 r}, \mu^{-1} s_{0}, \ldots, \mu^{-1} s_{l-1}\right) \text { if } 1 \leqslant l \leqslant r,
$$

and

$$
t_{2 l-2 r-2}=\mu^{2} \alpha\left(s_{l}, s_{l+1}, \ldots, s_{2 r}, \mu^{-1} s_{0}, \ldots, \mu^{-1} s_{l-1}\right) \text { if } r+1 \leqslant l \leqslant 2 r .
$$


Proof of Claim 2. Suppose $(s, t) \in X \cap \phi_{\theta^{l}}(X)$. There is an $(x, y) \in X$ such that $(s, t)=$ $\phi_{\theta^{l}}((x, y))$. From the equation $(s, t)=\left(\theta^{l} x, \theta^{2 l} y\right)$ we obtain by equating coefficients of $\theta^{0}, \theta^{1}, \ldots, \theta^{2 r}$ in the first component,

$$
x_{i}=s_{l+i} \text { for } 0 \leqslant i \leqslant 2 r-l \text { and } \mu x_{i}=s_{i-2 r+l-1} \text { for } 2 r-l+1 \leqslant i \leqslant 2 r .
$$

If $1 \leqslant l \leqslant r$, then we obtain $t_{2 l-1}=\mu y_{2 r}$ by considering the coefficient of $\theta^{2 l-1}$ in the second component. Similarly, if $r+1 \leqslant l \leqslant 2 r$, we obtain $t_{2 l-2 r-2}=\mu^{2} y_{2 r}$ by considering the coefficient of $\theta^{2 l-2 r-2}$ in the second component. Since $(x, y) \in X$, we have by Claim 1 that

$$
y_{2 r}=\alpha\left(x_{0}, x_{1}, \ldots, x_{2 r}\right) .
$$

Using (6), we can solve for the $x_{i}$ 's in terms of the $s_{j}$ 's and then substitute into (7) to complete the proof of Claim 2.

For $0 \leqslant k \leqslant 2 r$, let

$$
U_{k}=\{\{i, j\} \subset\{0,1, \ldots, 2 r\}: i+j \equiv k(\bmod 2 r+1)\} .
$$

Given $\{i, j\} \subset\{0,1, \ldots, 2 r\}$, let

$$
\mu_{\{i, j\}}= \begin{cases}1 & \text { if } 1 \leqslant i+j \leqslant 2 r \\ \mu & \text { if } 2 r+1 \leqslant i+j \leqslant 4 r-1\end{cases}
$$

Claim 3: Suppose $(s, t) \in Z$. If $1 \leqslant l \leqslant r$, then

$$
t_{2 l-1}=2 \mu s_{l+r}^{2}+4 \sum_{\{i, j\} \in U_{2 l-1}} \mu_{\{i, j\}} s_{i} s_{j} .
$$

If $0 \leqslant l \leqslant r-1$, then

$$
t_{2 l}=2 s_{l}^{2}+4 \sum_{\{i, j\} \in U_{2 l}} \mu_{\{i, j\}} s_{i} s_{j}
$$

Proof of Claim 3. First suppose $1 \leqslant l \leqslant r$. By Claim 2 ,

$$
t_{2 l-1}=\mu \alpha\left(s_{l}, s_{l+1}, \ldots, s_{2 r}, \mu^{-1} s_{0}, \ldots, \mu^{-1} s_{l-1}\right) .
$$

Using the definition of $\alpha$, we get that

$$
\begin{aligned}
t_{2 l-1} & =\mu\left(2 s_{l+r}^{2}+4\left(s_{l} \mu^{-1} s_{l-1}+s_{l+1} \mu^{-1} s_{l-2}+\cdots+s_{2 l-1} \mu^{-1} s_{0}\right.\right. \\
& \left.\left.+s_{2 l} s_{2 r}+\cdots+s_{l+r-1} s_{l+r+1}\right)\right) \\
& =2 \mu s_{l+r}^{2}+4 \sum_{\{i, j\} \in U_{2 l-1}} \mu_{\{i, j\}} s_{i} s_{j} .
\end{aligned}
$$

Assume now that $0 \leqslant l \leqslant r-1$. By Claim 2 ,

$$
t_{2 l}=\mu^{2} \alpha\left(s_{l+r+1}, s_{l+r+2}, \ldots, s_{2 r}, \mu^{-1} s_{0}, \ldots, \mu^{-1} s_{l+r}\right) .
$$

We can now proceed as before using the definition of $\alpha$. 
Claim 4: Let $s, x \in \mathbb{F}_{q^{t}}$. If $0 \leqslant l \leqslant 2 r$, then the coefficient of $\theta^{l}$ in $(s+x)^{2}$ is

$$
\left(s_{l / 2}+x_{l / 2}\right)^{2}+2 \sum_{\{i, j\} \in U_{l}} \mu_{\{i, j\}}\left(s_{i}+x_{i}\right)\left(s_{j}+x_{j}\right) \text { if } l \text { is even, }
$$

and

$$
\mu\left(s_{r+l / 2+1 / 2}+x_{r+l / 2+1 / 2}\right)^{2}+2 \sum_{\{i, j\} \in U_{l}} \mu_{\{i, j\}}\left(s_{i}+x_{i}\right)\left(s_{j}+x_{j}\right) \text { if } l \text { is odd }
$$

Proof of Claim 4. Consider

$$
(s+x)^{2}=\sum_{i=0}^{2 r}\left(s_{i}+x_{i}\right)^{2} \theta^{2 i}+2 \sum_{0 \leqslant i<j \leqslant 2 r}\left(s_{i}+x_{i}\right)\left(s_{j}+x_{j}\right) \theta^{i+j} .
$$

The claim follows from the definitions of $\mu_{\{i, j\}}, U_{l}$, and the identity $\theta^{2 r+k}=\mu \theta^{k-1}$ for $1 \leqslant k \leqslant 2 r$.

Claim 5: If $(s, t),(x, y) \in Z$ and $(s+x)^{2}=t+y$, then

$$
\mu\left(s_{l+r}-x_{l+r}\right)^{2}+2 \sum_{\{i, j\} \in U_{2 l-1}} \mu_{\{i, j\}}\left(s_{i}-x_{i}\right)\left(s_{j}-x_{j}\right)=0 \text { for } 1 \leqslant l \leqslant r,
$$

and

$$
\left(s_{l}-x_{l}\right)^{2}+2 \sum_{\{i, j\} \in U_{2 l}} \mu_{\{i, j\}}\left(s_{i}-x_{i}\right)\left(s_{j}-x_{j}\right)=0 \text { for } 0 \leqslant l \leqslant r .
$$

Proof of Claim 5. By Claim 4, equating coefficients of $1, \theta, \ldots, \theta^{2 r}$ in the equation $(s+$ $x)^{2}=t+y$ gives

$$
t_{2 l-1}+y_{2 l-1}=\mu\left(s_{l+r}+x_{l+r}\right)^{2}+2 \sum_{\{i, j\} \in U_{2 l-1}} \mu_{\{i, j\}}\left(s_{i}+x_{i}\right)\left(s_{j}+x_{j}\right) \text { if } 1 \leqslant l \leqslant r,
$$

and

$$
t_{2 l}+y_{2 l}=\left(s_{l}+x_{l}\right)^{2}+2 \sum_{\{i, j\} \in U_{2 l}} \mu_{\{i, j\}}\left(s_{i}+x_{i}\right)\left(s_{j}+x_{j}\right) \text { if } 0 \leqslant l \leqslant r .
$$

Now we apply Claim 3 to $t_{2 l-1}$ and $y_{2 l-1}$. This gives

$$
\begin{aligned}
& 2 \mu\left(s_{l+r}^{2}+x_{l+r}^{2}\right)+4 \sum_{\{i, j\} \in U_{2 l-1}} \mu_{\{i, j\}}\left(s_{i} s_{j}+x_{i} x_{j}\right)= \\
& \mu\left(s_{l+r}+x_{l+r}\right)^{2}+2 \sum_{\{i, j\} \in U_{2 l-1}} \mu_{\{i, j\}}\left(s_{i}+x_{i}\right)\left(s_{j}+x_{j}\right)
\end{aligned}
$$

for $1 \leqslant l \leqslant r$. This can be rewritten as

$$
\mu\left(s_{l+r}-x_{l+r}\right)^{2}+2 \sum_{\{i, j\} \in U_{2 l-1}} \mu_{\{i, j\}}\left(s_{i}-x_{i}\right)\left(s_{j}-x_{j}\right)=0 .
$$


A similar application of Claim 3 (and Claim 1 in the case of $t_{2 r}$ and $y_{2 r}$ ) gives

$$
\left(s_{l}-x_{l}\right)^{2}+2 \sum_{\{i, j\} \in U_{2 l}} \mu_{\{i, j\}}\left(s_{i}-x_{i}\right)\left(s_{j}-x_{j}\right)=0
$$

for $0 \leqslant l \leqslant r$.

We are now ready to find an upper bound on the maximum degree of the subgraph of $G_{q^{t}}$ induced by $Z$. Fix a vertex $(s, t) \in Z$. Suppose $(x, y)$ is a neighbor of $(s, t)$ with $(x, y) \in Z$. By Claim $5,\left(x_{0}, \ldots, x_{2 r}\right) \in \mathbb{F}_{q}^{2 r+1}$ is a solution to the system

$$
\mu\left(s_{l+r}-x_{l+r}\right)^{2}+2 \sum_{\{i, j\} \in U_{2 l-1}} \mu_{\{i, j\}}\left(s_{i}-x_{i}\right)\left(s_{j}-x_{j}\right)=0 \text { for } 1 \leqslant l \leqslant r,
$$

and

$$
\left(s_{l}-x_{l}\right)^{2}+2 \sum_{\{i, j\} \in U_{2 l}} \mu_{\{i, j\}}\left(s_{i}-x_{i}\right)\left(s_{j}-x_{j}\right)=0 \text { for } 0 \leqslant l \leqslant r .
$$

If we set $z_{i}=s_{i}-x_{i}$ for $0 \leqslant i \leqslant 2 r$, then we see that we have a solution to the following system of $2 r+1$ homogeneous quadratic equations in the $2 r+1$ unknowns $z_{0}, \ldots, z_{2 r+1}$ :

$$
\mu z_{l+r}^{2}+2 \sum_{\{i, j\} \in U_{2 l-1}} \mu_{\{i, j\}} z_{i} z_{j}=0 \text { for } 1 \leqslant l \leqslant r,
$$

and

$$
z_{l}^{2}+2 \sum_{\{i, j\} \in U_{2 l}} \mu_{\{i, j\}} z_{i} z_{j}=0 \text { for } 0 \leqslant l \leqslant r .
$$

Lemma 13. The number of solutions $\left(z_{0}, \ldots, z_{2 r}\right) \in \mathbb{F}_{q}^{2 r+1}$ to the above system of $2 r+1$ homogeneous quadratic equations is at most

$$
\frac{2 r+5}{3} q^{\frac{4 r}{3}+1}
$$

Proof. Let $m$ be the largest integer such that $m \leqslant \frac{2(r+1)}{3}$. If there is set $T$ of size $m$ such that each $z_{i}$ in the set $\left\{z_{i}: i \in T\right\}$ either is zero or is determined uniquely by the $z_{j}$ 's in the set $\left\{z_{j}: j \in\{0,1, \ldots, 2 r\} \backslash T\right\}$, then there are at most $q^{2 r+1-m}$ solutions. We will show there are at most $(m+1)$ choices for the index set $T$ which implies that we have at most $(m+1) q^{2 r+1-m}$ solutions in total.

Let $A$ be the $2(r-m+1) \times m$ matrix with entries in $\mathbb{F}_{q}$, where for $1 \leqslant i \leqslant 2(r-m+1)$ and $1 \leqslant j \leqslant m$, the $(i, j)$ entry of $A$ is the coefficient of $z_{j-1}$ in the equation

$$
\mu z_{m+r+(i-1) / 2}^{2}+2 \sum_{\{k, l\} \in U_{2(m+(i-1) / 2)-1}} \mu_{\{k, l\}} z_{k} z_{l}=0
$$

if $i$ is odd, and the coefficient of $z_{j-1}$ in the equation

$$
z_{m+(i-2) / 2}^{2}+2 \sum_{\{k, l\} \in U_{2(m+(i-2) / 2)}} \mu_{\{k, l\}} z_{k} z_{l}=0
$$


if $i$ is even. If $A$ is the matrix formed in this way, then one check that

$$
A=\left(\begin{array}{ccccc}
z_{2 m-1} & z_{2 m-2} & z_{2 m-3} & \ldots & z_{m} \\
z_{2 m} & z_{2 m-1} & z_{2 m-2} & \ldots & z_{m+1} \\
z_{2 m+1} & z_{2 m} & z_{2 m-1} & \ldots & z_{m+2} \\
\vdots & \vdots & \vdots & \ddots & \vdots \\
z_{2 r} & z_{2 r-1} & z_{2 r-2} & \ldots & z_{2 r-m+1}
\end{array}\right)
$$

As $m \leqslant \frac{2(r+1)}{3}$, we have $2(r-m+1) \geqslant m$. Let $x=\left(z_{0}, z_{1}, \ldots, z_{m-1}\right)^{T}$ and

$$
b_{i}= \begin{cases}-2^{-1} \mu z_{m+r+(i-1) / 2}-\sum_{\substack{\{k, l\} \in U_{2(m+(i-1) / 2)-1} \\\{k, l\} \cap\{0,1, \ldots, m-1\}=\emptyset}} \mu_{\{k, l\}} z_{k} z_{l} & \text { if } i \text { is odd } \\ -2^{-1} z_{m+(i-2) / 2}-\sum_{\substack{\{k, l\} \in U_{2(m+(i-2) / 2)} \\\{k, l\} \cap\{0,1, \ldots, m-1\}=\emptyset}} \mu_{\{k, l\}} z_{k} z_{l} & \text { if } i \text { is even }\end{cases}
$$

for $1 \leqslant i \leqslant 2(r-m+1)$. Let $b=\left(b_{1}, b_{2}, \ldots, b_{2(r-m+1)}\right)^{T}$.

We next show an upper bound $m+1$ for the possible choices for the index set $T$. Let $r_{i}$ be the $i$-th row of $A$ so that

$$
r_{i}=\left(z_{2 m-1+(i-1)}, z_{2 m-2+(i-1)}, z_{2 m-3+(i-1)}, \ldots, z_{m+(i-1)}\right) .
$$

If $r_{1}=\mathbf{0}$, then we take $T=\{m, m+1, \ldots, 2 m-1\}$. We assume $r_{1} \neq \mathbf{0}$ for the rest of the proof of the lemma.

Claim: If $i \geqslant 1$ and $r_{i+1} \in \operatorname{Span}_{\mathbb{F}_{q}}\left\{r_{1}, \ldots, r_{i}\right\}$, then

$$
z_{m+i+j} \in \operatorname{Span}_{\mathbb{F}_{q}}\left\{z_{m}, z_{m+1}, \ldots, z_{m+i-1}\right\} \text { for } j=0,1, \ldots, m-1 \text {. }
$$

Proof of Claim. We prove the claim by induction on $j$. Suppose

$$
r_{i+1}=\alpha_{1} r_{1}+\cdots+\alpha_{i} r_{i}
$$

for some $\alpha_{j} \in \mathbb{F}_{q}$. By considering the last coordinate, we get that

$$
z_{m+i}=\sum_{j=1}^{i} \alpha_{j} z_{m+(j-1)} \in \operatorname{Span}_{\mathbb{F}_{q}}\left\{z_{m}, \ldots, z_{m+i-1}\right\}
$$

establishing the base case $j=0$. If $z_{m+i+j_{0}} \in \operatorname{Span}_{\mathbb{F}_{q}}\left\{z_{m}, \ldots, z_{m+i-1}\right\}$ for $0 \leqslant j_{0} \leqslant m-2$, then by (8),

$$
z_{m+i+j_{0}+1}=\sum_{j=1}^{i} \alpha_{j} z_{m+j_{0}+1+(j-1)}=\alpha_{i} z_{m+i+j_{0}}+\sum_{j=1}^{i-1} \alpha_{j} z_{m+j+j_{0}} .
$$

By the inductive hypothesis, this is in $\operatorname{Span}_{\mathbb{F}_{q}}\left\{z_{m}, \ldots, z_{m+i-1}\right\}$. 
By the Claim, if there is an $i \in\{1,2, \ldots, m-1\}$ such that $r_{i+1} \in \operatorname{Span}_{\mathbb{F}_{q}}\left\{r_{1}, \ldots, r_{i}\right\}$, then there exist $m z_{i}$ 's that are uniquely determined by the other $z_{j}$ 's and we can take $T=\left\{z_{m+i}, z_{m+i+1}, \ldots, z_{2 m+i-1}\right\}$. Otherwise, $r_{1}, \ldots, r_{m}$ are linearly independent which implies that the rank of $A$ is at least $m$. It is at this step where we need $m \leqslant \frac{2(r+1)}{3}$ as we require the number of rows of $A$, which is $2(r-m+1)$, to be at least $m$. Since the rank of $A$ is at least $m$, there is at most one solution $x$ to $A x=b$. In this case we take $T=\{0,1, \ldots, m-1\}$ and each of $\left\{z_{0}, z_{1}, \ldots, z_{m-1}\right\}$ is determined by $\left\{z_{m}, z_{m+1}, \ldots, z_{2 r}\right\}$.

Altogether, we have at most

$$
(m+1) q^{\frac{4 r}{3}-\frac{1}{3}} \leqslant \frac{2 r+5}{3} q^{\frac{4 r}{3}+1}
$$

solutions which proves the lemma.

By Lemma 13, the maximum degree of $G_{q^{t}}[Z]$ is at most $\frac{2 r+5}{3} q^{\frac{4 r}{3}+1}$. Therefore,

$$
\chi\left(G_{q^{t}}\right) \leqslant \frac{2 r+5}{3} q^{\frac{4 r}{3}+1}+(2 r+1) q^{r+1}
$$

We obtain a coloring of $E R_{q^{t}}$ from a coloring of $G_{q^{t}}$ as before. We use one new color on the vertices $z_{1}, \ldots, z_{t}$, and then give $y$ any color that is used on $G_{q^{t}}$. This gives a coloring of $E R_{q^{t}}$ that uses at most $\frac{2 r+5}{3} q^{\frac{4 r}{3}+1}+(2 r+1) q^{r+1}+1$ colors which proves Theorem 2 .

\section{Proof of Theorem 7}

The following lemma is easily proved using the definition of adjacency in $G_{q}$.

Lemma 14. Suppose $\alpha_{i}, \alpha_{j}, \alpha_{k}$ are distinct elements of $\mathbb{F}_{q}$ such that

$$
\alpha_{i}+\alpha_{j}=a^{2}, \alpha_{j}+\alpha_{k}=b^{2}, \text { and } \alpha_{k}+\alpha_{i}=c^{2}
$$

for some $a, b, c \in \mathbb{F}_{q}$. If $x+y=a, y+z=b$, and $z+x=c$, then $\left\{\left(x, \alpha_{i}\right),\left(y, \alpha_{j}\right),\left(z, \alpha_{k}\right)\right\}$ induces a triangle in $G_{q}$.

Given an odd prime power $q$, let $\chi: \mathbb{F}_{q} \rightarrow\{0, \pm 1\}$ be the quadratic character on $\mathbb{F}_{q}$. That is, $\chi(0)=0, \chi(a)=1$ if $a$ is a nonzero square in $\mathbb{F}_{q}$, and $\chi(a)=-1$ otherwise. For the next lemma, we require some results on finite fields (see Chapter 5 of [15]).

Proposition 15. Let $q$ be an odd prime and $f(x)=a_{2} x^{2}+a_{1} x+a_{0} \in \mathbb{F}_{q}[x]$ where $a_{2} \neq 0$. If $a_{1}^{2}-4 a_{0} a_{2} \neq 0$, then

$$
\sum_{c \in \mathbb{F}_{q}} \chi(f(c))=-\chi\left(a_{2}\right)
$$


Proposition 16 (Weil). If $f(x) \in \mathbb{F}_{q}[x]$ is a degree $d \geqslant 1$ polynomial that is not the square of another polynomial, then

$$
\left|\sum_{x \in \mathbb{F}_{q}} \chi(f(x))\right| \leqslant(d-1) \sqrt{q} .
$$

Lemma 17. If $q>487$ is a power of an odd prime, then there are elements $\alpha_{1}, \ldots, \alpha_{5} \in \mathbb{F}_{q}^{*}$ such that $\alpha_{1}, \ldots, \alpha_{5}$ are all distinct, and

$$
\chi\left(\alpha_{i}+\alpha_{j}\right)=1
$$

for $1 \leqslant i<j \leqslant 5$.

Proof. Choose $\alpha_{1} \in \mathbb{F}_{q}^{*}$ arbitrarily. There are $\frac{q-1}{2}$ nonzero squares in $\mathbb{F}_{q}^{*}$ so we can easily find an $\alpha_{2} \in \mathbb{F}_{q} \backslash\left\{0, \alpha_{1}\right\}$ such that $\chi\left(\alpha_{1}+\alpha_{2}\right)=1$. Observe that this implies $\alpha_{2} \neq-\alpha_{1}$ otherwise $\chi\left(\alpha_{1}+\alpha_{2}\right)=0$. Assume that we have chosen $\alpha_{1}, \ldots, \alpha_{k} \in \mathbb{F}_{q}^{*}$ so that $\alpha_{1}, \ldots, \alpha_{k}$ are all distinct and

$$
\chi\left(\alpha_{i}+\alpha_{j}\right)=1
$$

for $1 \leqslant i<j \leqslant k$. Let

$$
f(x)=\prod_{i=1}^{k}\left(1+\chi\left(\alpha_{i}+x\right)\right)
$$

and $X=\left\{\beta \in \mathbb{F}_{q}: f(\beta)=2^{k}\right\}$. If $\beta \in \mathbb{F}_{q}$ and $f(\beta) \neq 0$, then $\chi\left(\alpha_{i}+\beta\right) \in\{0,1\}$ for $1 \leqslant i \leqslant k$. We have $\chi\left(\alpha_{i}+\beta\right)=0$ if and only if $\beta=-\alpha_{i}$. Therefore, there are at most $k$ distinct $\beta$ 's in $\mathbb{F}_{q}$ such that $0<f(\beta)<2^{k}$ which implies

$$
2^{k}|X|+k 2^{k-1} \geqslant \sum_{x \in \mathbb{F}_{q}} f(x)=q+\sum_{\emptyset \neq S \subset[k]} \sum_{x \in \mathbb{F}_{q}} \chi\left(\prod_{\alpha \in S}(x+\alpha)\right) .
$$

For any $i \in[k], \sum_{x \in \mathbb{F}_{q}} \chi\left(\alpha_{i}+x\right)=0$. For any $1 \leqslant i<j \leqslant k$,

$$
\sum_{x \in \mathbb{F}_{q}} \chi\left(x^{2}+\left(\alpha_{i}+\alpha_{j}\right) x+\alpha_{i} \alpha_{j}\right)=-1
$$

by Proposition 15 . When $k=2$, if $\frac{q-3-3 \cdot 2^{2}}{2^{3}} \geqslant 3+1$, then $|X| \geqslant 4$ and we can choose an $\alpha_{3} \in \mathbb{F}_{q} \backslash\left\{0, \alpha_{1}, \alpha_{2}\right\}$ that has the desired properties. When $k \in\{3,4\}$, we use Weyl's inequality to obtain a lower bound on $|X|$. Observe that since $\alpha_{1}, \ldots, \alpha_{k}$ are distinct, no product $\left(x+\alpha_{i_{1}}\right) \cdots\left(x+\alpha_{i_{l}}\right)$ for $1 \leqslant i_{1}<\cdots<i_{l} \leqslant k$ is the square of a polynomial in $\mathbb{F}_{q}[x]$. By Weyl's inequality, for any $1 \leqslant i_{1}<\cdots<i_{l} \leqslant k$,

$$
\left|\sum_{x \in \mathbb{F}_{q}} \chi\left(\left(x+\alpha_{i_{1}}\right) \cdots\left(x+\alpha_{i_{l}}\right)\right)\right| \leqslant l \sqrt{q} .
$$

The hypothesis on $q$ implies that $q$ is large enough so that $|X| \geqslant 5$ when $k=3$, and $|X| \geqslant 6$ when $k=4$. Therefore, we can inductively choose $\alpha_{4}$ and $\alpha_{5}$ so that $\alpha_{1}, \ldots, \alpha_{5}$ satisfy all of the required properties. 
Choose elements $\alpha_{1}, \ldots, \alpha_{5} \in \mathbb{F}_{q}^{*}$ satisfying the properties of Lemma 17. Let

$$
\alpha_{i}+\alpha_{j}=a_{i, j}^{2}
$$

for $1 \leqslant i<j \leqslant 5$. Since $\alpha_{i} \neq-\alpha_{j}$, no $a_{i, j}$ 's are zero. For $1 \leqslant i<j<k \leqslant 5$, let $x_{i, j, k}$, $y_{i, j, k}$, and $z_{i, j, k}$ be any elements of $\mathbb{F}_{q}$ that satisfy

$$
x_{i, j, k}+y_{i, j, k}=a_{i, j}, \quad y_{i, j, k}+z_{i, j, k}=a_{j, k}, \quad \text { and } z_{i, j, k}+x_{i, j, k}=a_{i, k} .
$$

Then the vertices $\left(x_{i, j, k}, \alpha_{i}\right),\left(y_{i, j, k}, \alpha_{j}\right)$, and $\left(z_{i, j, k}, \alpha_{k}\right)$ form a triangle in $G_{q}$ and this holds for any $1 \leqslant i<j<k \leqslant 5$.

Now we use these triangles, which are in $G_{q}$, together with the new vertices $z_{1}, \ldots, z_{q}, y$ that are added to $G_{q}$ to from $H_{q} \cong E R_{q}$ to obtain a subgraph with chromatic number at least four. For $1 \leqslant i \leqslant 5$, the vertex $z_{\alpha_{i}}$ is adjacent to all vertices of the form $\left(x, \alpha_{i}\right)$. The vertex $y$ is adjacent to each $z_{i}$. Consider the subgraph $H_{q}$ whose vertices are $y, z_{\alpha_{1}}, \ldots, z_{\alpha_{5}}$ together with all $\left(x_{i, j, k}, \alpha_{i}\right),\left(y_{i, j, k}, \alpha_{j}\right)$, and $\left(z_{i, j, k}, \alpha_{k}\right)$ for $1 \leqslant i<j<k \leqslant 5$. Suppose we have a proper 3 -coloring of this subgraph, say with colors 1,2 , and 3 . If the color 1 is given to three distinct vertices $z_{\alpha_{i}}$ 's, say $z_{\alpha_{i}}, z_{\alpha_{j}}$, and $z_{\alpha_{k}}$, then only colors 2 and 3 may be used on the triangle whose vertices are $\left(x_{i, j, k}, \alpha_{i}\right),\left(y_{i, j, k}, \alpha_{j}\right)$, and $\left(z_{i, j, k}, \alpha_{k}\right)$. Therefore, all three colors must be used to color the vertices in the set $\left\{z_{\alpha_{1}}, \ldots, z_{\alpha_{5}}\right\}$ but then no color may be used on $y$. The number of vertices in this subgraph is at most $1+5+\left(\begin{array}{l}5 \\ 3\end{array}\right) 3=36$.

\section{Concluding Remarks}

The upper bounds of Theorems 12 and 2 can be improved for large $q$ by applying the result of Alon et. al. [2] to the graph $G_{q^{t}}[Z]$ instead of using Brooks' Theorem. We have chosen to use Brooks' Theorem as then there is no issue of how large the implicit constant is, and because we believe that the upper bound should be closer to $O\left(q^{t / 2}\right)$.

Using a similar argument as the one used to prove Theorem 2, we can prove the following.

Theorem 18. If $q$ is a power of an odd prime, then

$$
\chi\left(G_{q^{3}}\right) \leqslant 6 q^{2} .
$$

A consequence is that $\chi\left(E R_{q^{3}}\right) \leqslant 6 q^{2}+1$ whenever $q$ is a power of an odd prime. Unfortunately, we were not able to extend this bound to the general case. In the $q^{3}$ case, one can explicitly compute the relations satisfied by vertices in $X$ (see Section 3.2) and then use these equations to bound the maximum degree of $G_{q^{3}}[X]$. Dealing with the set $X$ is one of the main obstacles in our approach.

We remark that if one could improve the bound in Lemma 13, it would improve our result in Theorem 2. It seems likely that Lemma 13 could be strengthened enough to improve the bound in Theorem 2 all the way down to

$$
\chi\left(E R_{q^{2 r+1}}\right) \leqslant(2 r+1+o(1)) q^{r+1} .
$$


Perhaps this can be done using techniques from algebraic geometry.

The conditions on $q$ for which there exists an irreducible polynomial $x^{t}-\mu \in \mathbb{F}_{q}[x]$ are known (see Theorem 3.75 of [15]). Let $q$ be a power of an odd prime and let $t \geqslant 3$ be an odd integer. Let $\operatorname{ord}(\mu, q)$ be the order of $\mu$ in group $\mathbb{F}_{q}^{*}$. Then $x^{t}-\mu \in \mathbb{F}_{q}[x]$ is irreducible if and only if each prime factor of $t$ divides ord $(\mu, q)$ but does not divide $\frac{q-1}{\operatorname{ord}(\mu, q)}$. Since $\mathbb{F}_{q}^{*}$ is cyclic, for any divisor $d$ of $q-1$, there is an element $a \in \mathbb{F}_{q}^{*}$ with $\operatorname{ord}(a, q)=d$. As long as $t$ divides $q-1$, we can choose an element $\mu \in \mathbb{F}_{q}^{*}$ so that $t$ divides $\operatorname{ord}(\mu, q)$ but $t$ does not divide $\frac{q-1}{\operatorname{ord}(\mu, q)}$. Therefore, if $q \equiv 1(\bmod t)$, then Theorem 2 applies and we obtain the upper bound $\chi\left(E R_{q^{t}}\right) \leqslant \frac{2 r+5}{3} q^{\frac{4 r}{3}+1}+(2 r+1) q^{r+1}+1$ in this case. For a fixed $t \geqslant 3$, Dirichlet's Theorem on primes in arithmetic progressions implies that there are infinitely many primes $p$ such that $p \equiv 1(\bmod t)$. Then for any $q$ which is an odd power of such $p$, we have $q \equiv 1(\bmod t)$.

The graph $E R_{q}$ is an example of an orthogonal polarity graph. Let $\Pi$ be a finite projective plane of order $q$ with point set $\mathcal{P}$ and line set $\mathcal{L}$. A polarity of $\Pi$ is a bijection $\pi: \mathcal{P} \cup \mathcal{L} \rightarrow \mathcal{P} \cup \mathcal{L}$ such that $\phi(\mathcal{P})=\mathcal{L}, \phi(\mathcal{L})=\mathcal{P}, \phi^{2}$ is the identity, and point $p$ is on line $l$ if and only if point $\phi(l)$ is on line $\phi(p)$. A point $p$ for which $p \in \phi(p)$ is called an absolute point. The polarity $\phi$ is called orthogonal if it has exactly $q+1$ absolute points. The corresponding orthogonal polarity graph is the graph with vertex set $\mathcal{P}$, and vertices $p_{1}$ and $p_{2}$ are adjacent if and only if $p_{1} \in \phi\left(p_{2}\right)$. The graph $E R_{q}$ is an orthogonal polarity graph which can be obtained from the projective plane $P G(2, q)$ and the polarity that sends the point $(x, y, z)$ to the line $[x, y, z]$, and sends the line $[x, y, z]$ to the point $(x, y, z)$ (loops in this graph must be removed to obtain $E R_{q}$ ). There are non-desaurgesian projective planes that have orthogonal polarities and this leads us to the following problem.

Problem 19. Determine if there is an absolute constant $C$ such that the following holds. If $G$ is an orthogonal polarity graph of a projective plane of order $q$, then

$$
\chi(G) \leqslant C q^{1 / 2} .
$$

\section{References}

[1] P. Allen, P. Keevash, B. Sudakov, J. Verstraëte, Turán numbers of bipartite graphs plus an odd cycle, J. Combin. Theory, Series B 106, (2014), 134-162.

[2] N. Alon, M. Krivelevich, B. Sudakov, Coloring graphs with sparse neighborhoods, J. Combin. Theory, Series B 77, (1999), 73-82.

[3] C. Balbuena, P. García-Vázquez, L. P. Montejano, Superconnectivity of graphs with odd girth $g$ and even girth $h$, Discrete Applied Mathematics 159 (2011), 91-99.

[4] W. G. Brown, On graphs that do not contain a Thomsen graph, Canada Math. Bull. 9, (1966), 281-289.

[5] J. Cilleruelo, Combinatorial problems in finite fields and Sidon sets, Combinatorica 32 (5) (2012), 497-511. 
[6] E. de Klerk, M. Newman, D. Pasechnik, R. Sotirov, On the Lovász $\theta$-number of almost regular graphs with application to Erdös-Rényi graphs, European J. Combin. 30, (2009), 879-888.

[7] P. Erdős, A. Rényi, V. T. Sós, On a problem of graph theory, Studia Sci. Math. Hungar. 1 (1966), 215-235.

[8] P. Erdős, M. Simonovits, Cube-saturated graphs and related problems, Progress in graph theory (Waterloo, Ont., 1982), 203-218, Academic Press, Toronto, 1984.

[9] Z. Füredi, Graphs without quadrilaterals, J. Comb. Theory, Series B 34, (1983), 187190.

[10] Z. Füredi, On the number of edges of quadrilateral-free graphs, J. Comb. Theory, Series B 68, (1996), 1-6.

[11] C. Godsil, M. Newman, Eigenvalue bounds for independent sets, J. Comb. Theory, Series B 98, (2008), 721-734.

[12] S. Hobart, J. Williford, The independence number for polarity graphs of even order planes, J. Algebr. Combin. 38, (2013), 57-64.

[13] A. Kostochka, P. Pudlák, V. Rödl, Some constructive bounds on Ramsey numbers, J. Comb. Theory, Series B 100, (2010), 439-445.

[14] F. Lazebnik, J. Verstraëte, On hypergraphs of girth five, Electronic J. of Combinatorics, 10, (2003), \#R25.

[15] R. Lidl, H. Niederreiter, Finite Fields, Cambridge University Press, 1997.

[16] D. Mubayi, J. Williford, On the independence number of the Erdös-Rényi and projective norm graphs and a related hypergraph, J. Graph Theory 56 (2007), no. 2, 113-127.

[17] J. Williford, Constructions in finite geometry with applications to graphs, Ph.D. thesis, University of Delaware (2004).

[18] L. A. Vinh, Graphs generated by Sidon sets and algebraic equations over finite fields, J. Comb. Theory, Series B 103, (2013), 651-657. 